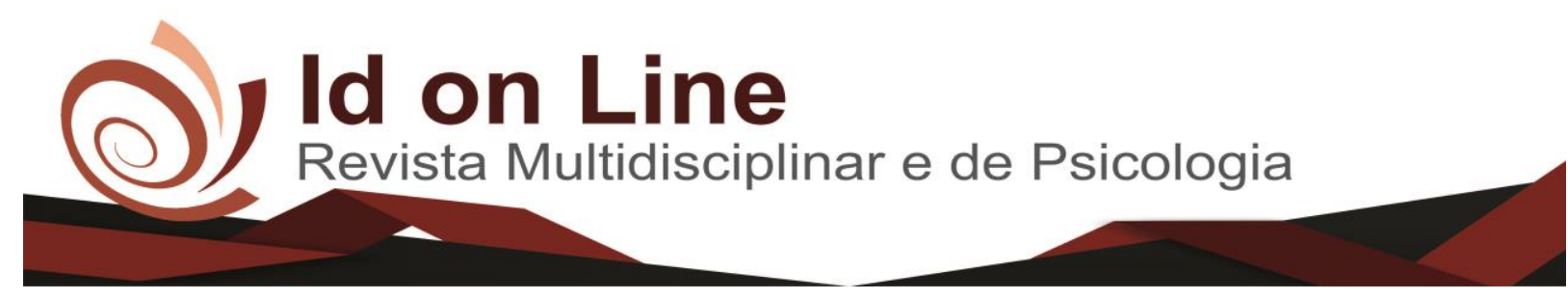

Artigo

\title{
Análise do Teor de Captopril em Farmácia de Manipulação no Município de Brumado
}

\author{
Natan Fernandes Pereira ${ }^{l}$; Caio Matheus da Rocha Couqueiro Monteiro de Oliveira ${ }^{2}$
}

Resumo: Com a expansão do setor magistral, houve um crescimento do investimento por parte dos seus proprietários, buscando um produto final de melhor qualidade. Neste trabalho será avaliado o teor de Captopril de farmácia magistral no município de Brumado, na Bahia. A escolha do Captopril, medicamento anti-hipertensivo, se deu por este ser o primeiro e mais conhecido inibidor da enzima conversora da Angiotensina (IECA). O presente estudo constitui-se em uma pesquisa experimental baseada na espectrofotometria de absorção molecular ultravioleta para determinação do teor de princípio ativo de captopril $25 \mathrm{mg}$ em formulações magistrais. Os resultados obtidos na pesquisa demonstra que as amostras analisadas encontram-se com o teor médio dentro do intervalo determinado de $90 \%$ a $110 \%$, sendo assim aptas para o consumo.

Palavras-chave: Espectrofotometria. Controle de qualidade. Hipertensão arterial.

\section{Analysis of the Captopril Content in a Manipulation Pharmacy in the Municipality of Brumado}

\begin{abstract}
With the expansion of the master sector, there was an increase in investment by its owners, seeking a better quality end product. In this work the Captopril content of master pharmacy will be evaluated in the municipality of Brumado, Bahia. The choice of Captopril, the antihypertensive drug, was the first and best known angiotensin converting enzyme inhibitor (ACEI). The present study is an experimental research based on the ultraviolet molecular absorption spectrophotometry for determining the active substance content of captopril 25 $\mathrm{mg}$ in magisterial formulations. The results obtained in the research show that the analyzed samples are with the average content within the given range of $90 \%$ to $110 \%$, thus being suitable for consumption.
\end{abstract}

Keywords: Spectrophotometry. Quality control. Arterial hypertension.

\section{Introdução}

Apesar da sombra da crise econômica que ronda a economia do país no ultimo ano, uma estimativa da IMS Health prevê um aumento de mais de 100\% no mercado farmacêutico para os próximos 05 anos, em decorrência do envelhecimento da população e crescente acesso a planos privados de saúde. Estima-se que o segmento alcance a marca dos R $\$ 87$ bilhões ainda em 2017 (FEBRAFAR, 2017).

\footnotetext{
${ }^{1}$ Graduando em Farmácia Generalista pela Faculdade Independente do Nordeste. Contato: natanfpl@hotmail.com;

${ }^{2}$ Farmacêutico Clínico-industrial graduado pela Universidade Estadual de Feira de Santana. Especialista em Análises Clínicas e Toxicológicas pela Faculdade Independente do Nordeste (FAINOR). Mestre em Química Analítica pela Universidade Estadual do Sudoeste da Bahia (UESB). Docente do curso de Farmácia na Faculdade Independente do Nordeste (FAINOR).
} 
O setor magistral não fica fora da conta e foi responsável pela movimentação de aproximadamente R\$ 5 bilhões somente em 2016, concentrando cerca de 7200 estabelecimentos ao redor do país, segundo "O Panorama Setorial Anfarmag: Farmácias de Manipulação Brasileiras - 2015/2016" demonstrou que 72\% dos proprietários de farmácias magistrais no país estão investindo em melhorias e $87 \%$ pretendem investir na farmácia nos próximos meses (CONSULFARMA, 2016).

Com a expansão do setor magistral há o aumento na necessidade de uma melhor qualidade do produto final, bem como de um maior rigor no controle de qualidade dos produtos farmacêuticos manipulados, visto que neste segmento, os avanços que ocorrem se dão por conta de alterações na legislação sanitária e em função das forças do mercado(BERNARDES; MUELLER; GEBARA, 2010).

O processo de manipulação de medicamentos magistrais gera uma crescente crítica por parte da população e setores de fiscalização como a ANVISA, embora se admita que a comparação com o controle de qualidade realizado no processo industrial, principalmente sob o produto final, torna-se inviável pela estrutura de uma farmácia magistral, visto que a mesma não tem condições de arcar com os equipamentos e logística necessários para tanto, sendo atualmente necessário, pela legislação, determina-se o cálculo de peso médio e coeficiente de variação de cápsulas manipuladas (BRASIL, 2005).

As principais medicações manipuladas são aquelas que se destinam para o tratamento de doenças crônicas entre elas se destaca a Hipertensão Arterial (HA), segundo Silva e Souza (2004) é uma doença sistêmica considerada uma síndrome com diversos fatores de origem e atualmente é um dos maiores problemas da saúde pública, sendo alimentação desregulada, rica em sódio e altos níveis de gordura, além do consumo de álcool e cigarro associados ao sedentarismo,os fatores que mais contribuem para o aumento dos níveis pressóricos e são considerados facilitadores para o início e desenvolvimento de demais doenças cardiovasculares em associação a HA (SOCIEDADE BRASILEIRA DE CARDIOLOGIA, 2016).

O Captopril é um dos medicamentos mais utilizado como anti-hipertensivo e vasodilatador na terapêutica da insuficiência cardíaca, sendo disponível em doses industriais de $12,5 \mathrm{mg}, 25 \mathrm{mg}$ e $50 \mathrm{mg}$, ele também é amplamente comercializado através de farmácias magistrais, e seu mecanismo de ação se dá através do bloqueio da enzima responsável pela conversão da Angiotensina I em Angiotensina II, essa última com ação vasoconstrictora (RANG, H.P.; DALE, 2016). 
Nessa perspectiva, o presente trabalho avaliará o teor de captopril de farmácia magistral do município de Brumado, na Bahia. O município possui população estimada em 69473 habitantes e uma farmácia de manipulação filiada a Associação Nacional de Farmácias Magistrais (ANFARMAG). Serão avaliadas cápsulas de captopril de 25 mg para determinação de teor através de espectrofotometria, de acordo com parâmetros da Farmacopeia Brasileira.

\section{Base Conceitual}

A Hipertensão Arterial (HA) é uma doença crônica não transmissível (DCNT) caracterizada por elevados níveis de pressão arterial com valores acima de 140 e/ou $90 \mathrm{mmHg}$ (SOCIEDADE BRASILEIRA DE CARDIOLOGIA, 2016). Considerada uma condição clínica multifatorial, ela está frequentemente associada a outras co-morbidades tais como alterações funcionais e distúrbios metabólicos, além de ser agravada pela presença de dislipidemia, obesidade abdominal, intolerância à glicose e diabetes mellitus (DM), considerados fatores de risco (FR).

Entre as DNCTs, a hipertensão, apesar de ser uma condição clínica tratável, é a responsável pela maior morbidade e mortalidade (MENGUE et al., 2016). É considerada a condição mais comumente observada na atenção primária e pode levar ao infarto do miocárdio, AVC, insuficiência renal e morte, se não for rapidamente detectada e tratada (JAMES et al., 2014).

No Brasil, a HA atinge aproximadamente 36 milhões de adultos, mais de $60 \%$ dos idosos, além de contribuir para 50\% das mortes por doença cardiovascular (DCV). (SOCIEDADE BRASILEIRA DE CARDIOLOGIA, 2016). Mesmo quando não tem consequências fatais, a hipertensão, juntamente com a diabetes mellitus, causa um grande impacto na vida dos portadores por ter uma característica clínica incapacitante, levando a perda da produtividade do trabalho queda da renda familiar bruta. Estima-se que, entre 2006 a 2015, essa perda chegou a US\$ 4,18 bilhões no Brasil (ABEGUNDE et al., 2007).

Estudos epidemiológicos apontam que o desenvolvimento da Hipertensão Arterial está associado ao avanço da idade (em ambos os sexos), excesso de peso, circunferência da cintura elevada (e classificação de IMC acima da média), fumo e consumo de bebida alcoólica. Desses, o excesso de peso é o fator mais preocupante, estando associado não só à HA, mas também a outras doenças cardiovasculares. (SILVEIRA et al., 2013). 
A farmacologia da HA conta, atualmente, com diversas classes de medicamentos e diferentes mecanismos de ação. As características do paciente hipertenso incluindo sexo, idade, presença ou não de outras doenças e estilo de vida são determinantes para a melhor abordagem do quadro clínico (HIGGINS; WILLIAMS, 2007). Existem 05 classes terapêuticas para o tratamento da Hipertensão: inibidores da ECA, bloqueadores de receptores de angiotensina, $\beta$ bloqueadores, bloqueadores de canal de cálcio, diuréticos tiazídicos (Tabela 2).

Tabela 1 - Dosagem Baseada em Evidencias para Medicamentos Anti-Hipertensivos.

\begin{tabular}{|c|c|c|c|}
\hline $\begin{array}{l}\text { Medicações Anti- } \\
\text { hipertensivas }\end{array}$ & $\begin{array}{l}\text { Dose inicial } \\
\text { diária (mg) }\end{array}$ & $\begin{array}{c}\text { Dose alvo nos } \\
\text { estudos revisados }\end{array}$ & $\begin{array}{c}\text { No. de doses } \\
\text { por dia }\end{array}$ \\
\hline \multicolumn{4}{|l|}{ Inibidores da ECA } \\
\hline Captopril & 50 & $150-200$ & 2 \\
\hline Enalapril & 5 & 20 & $1-2$ \\
\hline Lisinopril & 10 & 40 & 1 \\
\hline \multicolumn{4}{|c|}{$\begin{array}{c}\text { Bloqueadores de receptor de } \\
\text { angiotensina }\end{array}$} \\
\hline Eprosartana & 400 & $600-800$ & $1-2$ \\
\hline Candesartana & 4 & $12-32$ & 1 \\
\hline Losartana & 50 & 100 & $1-2$ \\
\hline Valsartana & $40-80$ & $160-320$ & 1 \\
\hline Irbesartana & 75 & 300 & 1 \\
\hline \multicolumn{4}{|l|}{$\beta$-bloqueadores } \\
\hline Atenolol & $25-50$ & 100 & 1 \\
\hline Metoprolol & 50 & $100-200$ & $1-2$ \\
\hline \multicolumn{4}{|c|}{$\begin{array}{l}\text { Bloqueadores de canal de } \\
\text { cálcio }\end{array}$} \\
\hline Anlodipino & 2.5 & 10 & 1 \\
\hline $\begin{array}{l}\text { Diltiazem liberação } \\
\text { prolongada }\end{array}$ & $120-180$ & 360 & 1 \\
\hline Nitrendipino & 10 & 20 & $1-2$ \\
\hline \multicolumn{4}{|l|}{ Diuréticos tipotiazídicos } \\
\hline Bendroflumetiazida & 5 & 10 & 1 \\
\hline Clortalidona & 12.5 & $12.5-25$ & 1 \\
\hline Hidroclorotiazida & $12.5-25$ & $25-100$ & $1-2$ \\
\hline Indapamida & 1.25 & $1.25-2.5$ & 1 \\
\hline
\end{tabular}

Fonte: adaptado de James et al., 2014 
Os Inibidores da Enzima Conversora de Angiotensina (IECAs) atuam na regulação da pressão arterial através do Sistema renina-angiotensina-aldosterona (SRAA) inibindo a enzima conversora de angiotensina I e impedindo a transformação de angiotensina I em angiotensina II, de ação vasoconstrictora. O primeiro IECA a entrar no mercado farmacêutico foi o Captopril e ainda hoje o mesmo é considerado um anti-hipertensivo eficaz e seguro com redução na morbimortalidade de doenças cardiovasculares. Os inibidores de ECA mais recentes, tais como Enalapril e Lisinopril, possuem maior meia vida e, consequentemente, duração de ação mais prologada (SOCIEDADE BRASILEIRA DE CARDIOLOGIA, 2016; RANG, H.P.; DALE, 2016). Os principais representantes da classe, Captopril e Enalapril, constam na Relação Nacional de Medicamentos Essenciais (RENAME) devido à eficácia clínica, segurança comprovada e custo-efetividade (OLIVEIRA et al., 2010)

Além da aplicação clínica na Hipertensão Arterial, os inibidores da ECA também são utilizados em outras doenças cardiovasculares tais como a insuficiência cardíaca, no pós-infarto do miocárdio (OLIVEIRA et al., 2011), em indivíduos de alto risco de cardiopatia além de uso em casos de nefropatia diabética e insuficiência renal progressiva.

Devido à sua alta confiabilidade e eficácia, ambos Captopril e Enalapril são amplamente utilizados no tratamento de hipertensão arterial e insuficiência cardíaca. Em um estudo fármaco epidemiológico descritivo, transversal e retrospectivo realizado na Secretaria Municipal da Saúde de Ribeirão Preto (SP), foram identificados 9560 pacientes usuários de IECA, sendo que $46,57 \%$ usavam captopril, 45,74\% usavam enalapril e 7,69\% utilizavam ambos, simultaneamente ou não, concluindo que há uma preferência de prescrição de inibidores da ECA em unidades do SUS devido à sua relevância terapêutica para o tratamento de DCV (OLIVEIRA et al., 2010).

De maneira similar, Longo e colaboradores (2011) realizaram análise farmacológica das principais classes dos agentes anti-hipertensivos utilizados pelos pacientes integrantes do Instituto Bairral de Psiquiatria em Itapira, SP. A amostra contou com 51 pacientes analisados e os inibidores da ECA foram os medicamentos mais utilizados na Unidade, em especial o Captopril. Quando observados pacientes com associação de medicamentos, os diuréticos tiazídicos foram os medicamentos mais prescritos juntos com os IECA(LONGO; MARTELLI; ZIMMERMANN, 2011).

A farmácia magistral está presente na história da farmácia desde o princípio, sendo a único método de desenvolvimento de medicamentos nos tempos antigos. No Brasil, eram chamadas de Boticas as primeiras farmácias de manipulação. As mesmas eram comandadas 
pelos boticários e oficiais de botica, que deveriam ter sua capacidade atestada pelo comissário do Físico-mor, além da verificação de cartas de licença.

Atualmente, as farmácias de manipulação e os medicamentos manipulados são regidos pelas recomendações previstas na Resolução de Diretoria Colegiada (RDC) 67/07 da Agencia Nacional de Vigilância Sanitária (ANVISA) (BRASIL, 2007).

A RDC 67/07 define Farmácia de Manipulação como um estabelecimento que produz fórmulas magistrais e oficinais. Nela, o farmacêutico, é o profissional habilitado a conhecer as formas farmacêuticas, a manipular as matérias-primas com técnica correta para se conseguir um medicamento desejado, além de ser responsável pela garantia e implementação da qualidade (BRASIL, 2007).

Apesar da existência de normas de boas práticas farmacêuticas e fiscalização da ANVISA, acidentes envolvendo superdosagens de medicamentos farmacêuticos manipulados foram responsáveis por diversos obtidos ao longo dos anos, prejudicando a imagem dos medicamentos manipulados, além de demonstrar uma maior necessidade de processos de controle de qualidade dentro de Farmácias Magistrais no país. Em 2004, um senhor de 67 anos foi internado em um hospital e, após 4 horas, veio a óbito. Após investigação, descobriu-se que o mesmo havia iniciado tratamento com cápsulas manipuladas de colchicina $60 \mathrm{mg} / \mathrm{dose}$. A colchicina é um medicamento exatamente tóxico, com dose máxima de 1,0 mg por unidade posológica (GABRIEL et al., 2014). Em 2007, um senhor de 70 anos foi a óbito pela ingestão de cápsulas de colchicina manipuladas com dose 100 vezes superior a dose máxima permitida (YANO; BUGNO; AURICCHIO, 2008).

\section{Metodologia}

O método utilizado para realizações dos testes utilizou como base a farmacopeia brasileira 2010 com adaptações, logo o protocolo foi o seguinte, preparo da solução padrão (estoque) de $1000 \mathrm{ppm}$ em recipiente de 0,5 L, pesou 0,5 g de captopril com elevado teor de pureza, em um béquer, homogeneizou com ácido clorídrico de concentração $0,1 \mathrm{~mol} / \mathrm{L}$ e transferiu para um balão volumétrico de $0,5 \mathrm{~L}$ e avoluma com o mesmo ácido clorídrico 0,1 mol/L formando solução de 1000 ppm.

A curva de calibração possuiseis pontos com as seguintes concentrações: 5,10,15,20,25,30,35 e $40 \mathrm{mg} / \mathrm{L}$.No preparo das soluções padrões pipetou $500 \mu 1(5 \mathrm{mg} / \mathrm{l}), 1000 \mu \mathrm{l}$ 
$(10 \mathrm{mg} / \mathrm{L}), \quad 1500 \mu \mathrm{l}(15 \mathrm{mg} / \mathrm{L}), 2000 \mu \mathrm{l}(20 \mathrm{mg} / \mathrm{L})$, usando micropipeta de $500 \mu 1,2500 \mu 1$

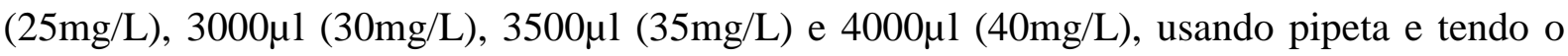
cuidado de completar o volume do balão volumétrico de 100,00 ml com ácido clorídrico 0,1 mol/L usando pipeta de Pasteur.

Usando o comprimento de onda em 212nm, específico para análise espectrofotométrica do captopril, mediu primeiro a absorbância da solução sem o analito, somente com o diluente ácido clorídrico $0,1 \mathrm{~mol} / \mathrm{L}$ sendo o branco, e depois mediu a absorbância de cada solução padrão e com esses dados encontra a curva de calibração e equação da reta. Como a curva de calibração vai de 10 a $40 \mathrm{ppm}$. Abriu as cápsulas da amostra de captopril num béquer de $50 \mathrm{ml}$, homogeneizou com ácido clorídrico $0,1 \mathrm{~mol} / \mathrm{Le}$ transferiu para balão volumétrico de $250 \mathrm{ml}$, depois filtrou em bomba de vácuo para remover excipiente e transferiu o filtrado para um béquer de $250 \mathrm{ml}$. Desse béquer pipetou $10 \mathrm{ml}$ da solução e colocou num balão volumétrico de $50 \mathrm{ml}$ para proceder à diluição e dessas faz a leitura da absorbância no espectrofotômetro.

\section{Resultados e Discussão}

Segundo a farmacopeia brasileira $5^{\text {a }}$ edição de 2010 o medicamento deve conter um teor mínimo de 90 até o limite de $110 \%$ analisa

da pela média do teste realizado, o local de estudo atende a mesma, entretanto se observamos cada teste isolado não existe uma uniformidade na concentração oscilando de valores abaixo do permitido e com uma variação na mesma de 89,40\% e 93,27\% conforme podemos observar na tabela 1 .

Tabela 2 - Resultado de analise de amostras do captopril.

\begin{tabular}{c|c|c|c|c|c}
\hline Amostra & Absorbância & $\begin{array}{c}{[\text { ] = mg/L }} \\
\text { (solução diluída) }\end{array}$ & $\begin{array}{c}{[\text { ] = mg/L }} \\
\text { (solução concentrada) }\end{array}$ & Massa de captopril (mg) & \% \\
\hline I & 0,411 & 18,41667 & 92,08333 & 23,02083 & 92,08 \\
\hline II & 0,402 & 17,88095 & 89,40476 & 22,35119 & 89,40 \\
\hline III & 0,415 & 18,65476 & 93,27381 & 23,31845 & 93,27 \\
\hline
\end{tabular}

Fonte: Dados da Análise.

$\mathrm{Na}$ perspectiva similar ao conduzida neste estudo, diversos trabalhos foram desenvolvidos com objetivo de conhecer o perfil de qualidade das cápsulas preparadas em Farmácias Magistrais, no analisando uniformidade de conteúdo e teor. Pissato e colaboradores 
(2006) avaliaram a qualidade de cápsulas de cloridrato de fluoxetina produzidas farmácias magistrais de Santa Catarina através de variação de peso, doseamento e uniformidade de conteúdo e comparam os resultados com análise do medicamento de referência e do medicamento distribuído pelo SUS. As formulações magistrais apresentaram-se dentro dos limites para as análises de variação de peso e teor de substância ativa. Entretanto, as mesmas foram reprovadas em testes de uniformidade de conteúdo (PISSATTO et al., 2006).

Ainda em 2006, Zarbielli et al., realizaram análise quantitativa e qualitativa de cápsulas de piroxicam manipuladas em farmácias no Rio Grande do Sul. As análises foram feitas por determinação do peso médio, da faixa de fusão, análise cromatográfica (CCD), espectrofotométrica na região do ultravioleta, teste de dissolução e teste de desintegração. No tocante às análises qualitativas, as amostras se mostraram dentro da faixa de variação permitida, entretanto, os resultados quantitativos referentes ao teor de piroxicam das duas farmácias magistrais foram inferiores ao especificado (ZARBIELLI; MACEDO; MENDEZ, 2006).

Em 2010, Markman e colaboradores avaliaram a qualidade de cápsulas de sinvastatina produzidas por farmácias magistrais de São Paulo, Guarulhos, São Bernardo do Campo e Campinas. O estudo contou com 18 amostras analisadas por HPLC e demais análises descritas na Farmacopeia Brasileira. De acordo com os autores, 04 amostras apresentaram variação de peso médio acima do permitido. No tocante ao teor de sinvastatina, 11 amostras foram aprovadas; em seis amostras, houve variação de $4 \%$ e $87 \%$ do valor declarado. No teste de UC, 15 amostras apresentaram valores abaixo de $85 \%$ e desvios-padrões maiores que $6 \%$. Por fim, no ensaio de dissolução, oito amostras foram reprovadas. Os ensaios apontam a falta de qualidade de cápsulas de sinvastatina produzidas por algumas farmácias de manipulação da região (MARKMAN; ROSA; KOSCHTSCHAK, 2010).

Couto e Tavares (2011) realizaram analise do perfil dos resultados de UC de capsulas de baixa dosagem feitas por um Laboratório de Controle de Qualidade que auxilia as farmácias magistrais de Santa Catarina. Eles observaram que 78,6\% das análises foram aprovadas, sendo o maior índice de reprovação às análises de teor de ativo fora do limite de $85-115 \%$, com até 83,6\% das amostras abaixo do especificado no rótulo do medicamento vendido (COUTO; TAVARES, 2011).

Dois estudos avaliaram a qualidade de cápsulas de captopril produzidas em farmácias magistrais. Marcatto e colaboradores (2005) avaliaram a qualidade de cápsulas de captopril de Farmácias Magistrais no Vale do Itajaí e Santa Catarina. Foram realizados testes de peso médio, teor de captopril e limite de dissulfeto de captopril, em comparação com padrão SQR. Apesar 
de 50\% das amostras magistrais adquiridas não passarem no teste de peso médio, todas foram aprovadas nos demais testes (MARCATTO et al., 2005). Visando o amplo desenvolvimento de diferentes formulações para uma mesma forma farmacêutica (cápsulas), Bernardes et al., desenvolveram e aplicaram testes de controle de qualidade à cápsulas de captopril $25 \mathrm{mg}$ contendo diferentes excipientes. Foram realizados testes de peso médio, dissolução, UC e doseamento por espectrofotômetro de acordo com parâmetros da Farmacopéia Brasileira. Apesar dos resultados diferirem entre as três formulações desenvolvidas, todas foram aprovadas nos testes com resultados dentro da faixa limite aceitáveis (BERNARDES; MUELLER; GEBARA, 2010).

\section{Conclusão}

Consideramos baseados nos resultados obtidos na pesquisa e na literatura utilizada, que as amostras de Captopril analisadas encontram-se com o teor médio dentro do intervalo determinado de $90 \%$ a $110 \%$, sendo assim aptas para o consumo. A metodologia utilizada é simples, mas de grande importância no controle de qualidade dos medicamentos produzidos em uma farmácia de manipulação, pois assegura ao consumidor final que o medicamento consumido encontra-se dentro dos parâmetros definidos pela legislação.

Vale salientar a importância de uma farmácia de manipulação, pois somente esta é capaz de atender de forma específica a necessidade de cada paciente, sendo necessário sempre aprimorar o controle de qualidade, assegurando assim uma garantia na qualidade do seu produto final.

\section{Referências}

ABEGUNDE, D. O. et al. The burden and costs of chronic diseases in low-income and middle-income countries. Lancet, v. 370, n. 9603, p. 1929-1938, 2007.

Agência Nacional de Vigilância Sanitária. Farmacopéia Brasileira. Volume 2, $5^{\text {a }}$ Edição, Brasília, 2010. Disponível em:

<http://www.anvisa.gov.br/hotsite/cd_farmacopeia/pdf/volume\%201.pdf > 
BERNARDES, M. L. P.; MUELLER, A.; GEBARA, K. S. Desenvolvimento e controle de qualidade de cápsulas contendo captopril 25 mg. Interbio - UNIGRAN, v. 4, n. 1, p. 15-21, 2010.

BRASIL. Agência Nacional de Vigilância Sanitária. Resolução - RDC no 67, de 8 de outubro de 2007. Regulamento técnico que institui as boas práticas de manipulação de Preparações Magistrais e Oficinais para Uso Humano em farmácias. Diário Oficial da União, Brasília, no 195, seção 1, p.29-58, 9 out. 2007

BRASIL. Subsídios à discussão sobre a proposta de regulamentação para farmácias magistrais. Revista de Saude Publica, v. 39, n. 4, p. 691-4, 2005.

COUTO, A. G.; TAVARES, R. C. Análise do perfil dos resultados de uniformidade de conteúdo de cápsulas de baixa dosagem produzidas em farmácias de manipulação de Santa Catarina. Revista de Ciencias Farmaceuticas Basica e Aplicada, v. 32, n. 2, p. 263-268, 2011.

FEBRAFAR, IMS: MERCADO FARMACÊUTICO DEVE ATINGIR R\$ 87 BILHÕES EM 2017. Maio, 2017. Disponível em:

<http://febrafar.com.br/ims-mercado-farmaceutico-deve-atingir-r-87-bilhoes-em-2017/>. Acesso em 22 de Maio de 2017.

GABRIEL, P. M. K. . et al. Colchicina: intoxicação e óbito, relato de caso. Visão Acadêmica, v. 24, n. 1, p. 189-197, 2014.

HIGGINS, B.; WILLIAMS, B. Pharmacological management of hypertension. Clinical medicine (London, England), v. 7, n. 6, p. 612-6, 2007.

JAMES, P. A. et al. 2014 Evidence-Based Guideline for the Management of High Blood Pressure in Adults. Jama, v. 311, n. 5, p. 507, 2014.

LONGO, M. A. T.; MARTELli, A.; ZIMMERMANN, A. Hipertensão arterial sistêmica: aspectos clínicos e análise farmacológica no tratamento dos pacientes de um setor de psicogeriatria do Instituto Bairral de Psiquiatria, no município de Itapira, SP. Revista Brasileira de Geriatria e Gerontologia, v. 14, n. 2, p. 271-284, 2011.

MARCATTO, A. P. et al. Análise de cápsulas de captopril manipuladas em farmácias. Revista de Ciencias Farmaceuticas Basica e Aplicada, v. 26, n. 3, p. 221-225, 2005.

MARKMAN, B. E. O.; ROSA, P. C. P.; KOSCHTSCHAK, M. R. W. Avaliação da qualidade de cápsulas de sinvastatina de farmácias magistrais. Revista de Saúde Pública, v. 44, n. 6, p. 1055-1062, 2010.

MENGUE, S. S. et al. Access to and use of high blood pressure medications in Brazil. Revista de Saude Publica, v. 50, n. supl 2, p. 1-9, 2016.

OLIVEIRA, C. M. X. et al. Padrões de Prescrição de Inibidores da Enzima Conversora da Angiotensina para Usuários do Sistema Único de Saúde. v. 23, n. 3, p. 171-177, 2010. 
OLIVEIRA, U. O. DE et al. Efeitos da Angiotensina-I e Isquemia na Recuperação Funcional em Corações Isolados. Arquivos Brasileiros de Cardiologia, v. 97, p. 390-396, 2011.

PISSATTO, S. et al. Avaliação da qualidade de cápsulas de cloridrato de fluoxetina. Acta Farmaceutica Bonaerense, v. 25, n. 4, p. 550-554, 2006.

RANG, H.P.; DALE, M. M. RANG \& DALE Farmacologia. 8. ed. Rio de Janeiro: Elsevier, 2016.

SILVA, J. L. L. DA; SOUZA, S. L. DE. Fatores de risco para hipertensão arterial sistêmica versus estilo de vida docente. Revista eletrônica de enfermagem :Faculdade de Enfermagem da Universidade Federal de Goiás., v. 6, n. 3, p. 1-10, 2004.

SILVEIRA, J. et al. Fatores associados à hipertensão arterial sistêmica e ao estado nutricional de hipertensos inscritos no programa Hiperdia status of hypertensive enrolled in the program Hiperdia. Cad. Saúde Coletiva, v. 21, n. 2, p. 129-134, 2013.

SOCIEDADE BRASILEIRA DE CARDIOLOGIA. $7^{\text {a }}$ Diretriz Brasileira De Hipertensão Arterial. v. 107, n. 3, 2016.

YANO, H. M.; BUGNO, A.; AURICCHIO, M. T. Intoxicação por colchicina em formulação manipulada Intoxication by Colchicine in a compounded pharmaceutical formulation. Rev. Inst. Adolfo Lutz, v. 67, n. 67, p. 234-236, 2008.

ZARBIELLI, M. G.; MACEDO, S.; MENDEZ, A. L. Controle de qualidade de cápsulas de piroxicam manipuladas em farmácias do município de Erechim - RS. Revista Brasileira de Farmácia, v. v.87, n. 2, p. 55-59, 2006.

\section{Como citar este artigo (Formato ABNT):}

PEREIRA, Natan F.; OLIVEIRA, Caio M. da R.C.M. Análise do Teor de Captopril em Farmácia de Manipulação no Município de Brumado. Id on Line Revista Multidisciplinar e de Psicologia, 2017, vol.11, n.38, p. 408-418. ISSN: 1981-1179.

Recebido: 30.10 .2017

Aceito: 01.11.2017 